ARTICLE

\title{
Notch-independent RBPJ controls angiogenesis in the adult heart
}

\author{
Ramón Díaz-Trelles ${ }^{1,2}$, Maria Cecilia Scimia1,2,3,†, Paul Bushway ${ }^{1,2}$, Danh Tran, Anna Monosov, \\ Edward Monosov ${ }^{1}$, Kirk Peterson ${ }^{3}$, Stacey Rentschler ${ }^{4}$, Pedro Cabrales ${ }^{2}$, Pilar Ruiz-Lozano ${ }^{5}$ \& Mark Mercola ${ }^{1,2,6}$
}

Increasing angiogenesis has long been considered a therapeutic target for improving heart function after injury such as acute myocardial infarction. However, gene, protein and cell therapies to increase microvascularization have not been successful, most likely because the studies failed to achieve regulated and concerted expression of pro-angiogenic and angiostatic factors needed to produce functional microvasculature. Here, we report that the transcription factor RBPJ is a homoeostatic repressor of multiple pro-angiogenic and angiostatic factor genes in cardiomyocytes. RBPJ controls angiogenic factor gene expression independently of Notch by antagonizing the activity of hypoxia-inducible factors (HIFs). In contrast to previous strategies, the cardiomyocyte-specific deletion of Rbpj increased microvascularization of the heart without adversely affecting cardiac structure or function even into old age. Furthermore, the loss of RBPJ in cardiomyocytes increased hypoxia tolerance, improved heart function and decreased pathological remodelling after myocardial infarction, suggesting that inhibiting RBPJ might be therapeutic for ischaemic injury.

\footnotetext{
${ }^{1}$ Sanford Burnham Prebys Medical Discovery Institute, La Jolla, California 92037, USA. ${ }^{2}$ Department of Bioengineering, Jacobs School of Engineering, University of California, San Diego, La Jolla, California 92093 USA. ${ }^{3}$ Division of Cardiology, Department of Medicine, University of California, San Diego, La Jolla, California 92093 USA. ${ }^{4}$ Departments of Medicine, Developmental Biology and Biomedical Engineering, Washington University, St Louis, Missouri 63110 USA. ${ }^{5}$ Department of Pediatrics, Stanford University, Stanford, California 94305 USA. ${ }^{6}$ Stanford Cardiovascular Institute and Department of Medicine, Stanford University, Stanford, California 94305, USA. † Present Address: Takeda Pharmaceuticals, Cambridge, Massachusetts 02139 USA. Correspondence and requests for materials should be addressed to R.D-T. (email: trelles@sbpdiscovery.org) or to M.M. (email: mmercola@stanford.edu).
} 
A ngiogenesis in the adult heart is tightly regulated in order to maintain cardiac function in response to workload ${ }^{1}$. The mechanisms that coordinate angiogenesis and workload are poorly understood, but involve the regulated secretion of angiogenic factors from cardiomyocytes ${ }^{2,3}$.

Increasing angiogenesis is of therapeutic interest to treat chronic myocardial ischaemia, ${ }^{4,5}$ as well as acutely after myocardial infarction since many patients have inadequate perfusion despite restoration of coronary artery blood flow ${ }^{6}$. Despite pre-clinical proof of concept, clinical trials of single factor, gene and cell-based therapies have provided inconsistent results, and identifying a definitive means of inducing clinically useful therapeutic angiogenesis remains elusive ${ }^{4-6}$. A challenge in achieving therapeutic angiogenesis is that the formation of functional vessels involves the coordinated activities of numerous factors, some of which promote endothelial cell proliferation and vessel sprouting (for example, vascular endothelial growth factor (VEGF) and fibroblast growth factor (FGF)), while others drive vessel remodelling and maturation (for example, angiopoietins) ${ }^{7}$.

Since pressure overload increases myocardial angiogenesis and activates Notch signalling ${ }^{8,9}$, we hypothesized that Notch and its transcriptional effector RBPJ (recombination signal binding protein for immunoglobulin kappa J region) might function in cardiomyocytes as a high level modulator of myocardial angiogenesis. RBPJ is ubiquitously expressed, and can either activate genes by forming a complex with the Notch intracellular domain (NICD) when Notch is active ${ }^{10}$, or silence an overlapping but non-identical set of genes by recruiting co-repressors in the absence of Notch signalling ${ }^{11-13}$.

Our studies revealed that RBPJ represses the production of pro-angiogenic and angiostatic factors in cardiomyocytes of the unstressed heart, such that the adult cardiomyocyte (ACM)specific deletion of rbpj increases microvessel density, confers physiological tolerance to hypoxia and protects the heart from ischaemic injury. Contrary to expectations, activation of Notch itself did not induce an angiogenic programme. We present evidence that RBPJ controls angiogenic factor gene expression by direct binding and inhibition of HIF $1 \alpha$ and $2 \alpha$. Thus, RBPJ has a novel Notch-independent role as a repressor of hypoxia responsiveness and myocardial angiogenesis.

\section{Results}

RBPJ acts as a transcriptional repressor in healthy ACM. We first investigated the location and activity of RBPJ in the mouse adult heart. Nuclear-localized and faint cytoplasmic RBPJ is readily detected in the adult heart, both in cardiomyocytes and non-myocytes, and its level does not change substantially following either chronic pressure overload by thoracic aortic constriction (TAC) or myocardial infarction (Fig. 1a,b; Supplementary Fig. 1). In contrast, RBPJ was nearly undetectable basally by immunostaining with the monoclonal antibody T6709 (ref. 14) (Fig. 1d), but was strikingly evident in response to a doxycyclin-inducible, transgenic NICD (iNICD) ${ }^{15}$ (Fig. 1c,f) and after TAC or myocardial infarction (MI) (Fig. 1e; Supplementary Fig. 1c-f) coinciding with elevated expression of Notch ligands (Supplementary Fig. 2a-d). T6709-detection correlates with Notch activation ${ }^{14}$, suggesting that RBPJ in the heart might repress gene expression basally and mediate Notch signalling after MI and pressure overload.

To investigate the potential function of RBPJ in the heart, we deleted Rbpj in ACM with Myl2-Cre (cKO). cKO mice had normal viability consistent with post-natal deletion (Supplementary Table 1; Supplementary Fig. 3a,b). Deletion increased expression of the endogenous Notch/RBPJ target genes Hes 1 and Hey2 identically to the effect of inducing Notch activation (iNICD) in unstressed hearts (Fig. 1g,h), and increased the content of activating tri-methylated (K4) histone-H3 $\left(\mathrm{H} 3 \mathrm{~K} 4 \mathrm{me}^{3}\right)$ in a proximal region of the endogenous Hes 1 and Hey2 promoter containing RBPJ-binding motifs (Fig. 1i). In vitro, RBPJ overexpression repressed a Hes1 promoter-luciferase reporter (Fig. 1j). Finally, Hes1 messenger RNA (mRNA) content was higher in isolated cKO relative to wild-type (WT) cardiomyocytes, and could be induced by adenoviral delivery of NICD in an RBPJ-dependent manner (Fig. 1k). These data indicate that RBPJ acts as a transcription repressor basally in cardiomyocytes, but is converted to an activator upon pressure overload or MI (Fig. 11).

Lack of RBPJ induces angiogenesis in the heart. Next, we evaluated the consequences of rbpj deletion. cKO hearts showed no evidence of cardiomyocyte pathology (see Supplementary Fig. 4 for a detailed phenotypic characterization), and responded normally to pressure overload for all parameters analyzed, including cardiac function (Supplementary Table 2) and hypertrophy (Supplementary Fig 5). However, the loss of RBPJ significantly increased the number of capillaries relative to cardiomyocytes (CC ratio) from $1.6 \pm 0.27$ in WT hearts to $2.1 \pm 0.28$ in cKO hearts (Fig. 2a-c). cKO capillaries were morphologically normal even into old age (20 months, Fig. 2d,e), and were perfused by ILB4 lectin to the same degree as WT capillaries (Fig. 2f,g). Fibrinogen was not detected outside the vessel walls (Fig. $2 \mathrm{~h}-\mathrm{k}$ ), indicating that the capillaries were not leaky, in contrast to capillaries following myocardial infarction as a control (Fig. 2j,k), or following chronic hypoxia-inducible factor (HIF) stabilization ${ }^{16,17}$. Thus, deletion of $r b p j$ in cardiomyocytes increased the density of functional capillaries without inducing cardiac pathology.

Capillary density normally increases in response to chronically elevated blood pressure. In WT mice, 2 weeks of TAC significantly increased the CC ratio to $2.2 \pm 0.33$. Myocardial microvascularization did not increase after TAC in the cKO-RBPJ mice beyond the already elevated basal level $(1.9 \pm 0.17 \mathrm{CC}$ ratio, statistically indistinguishable from the basal level). One possible explanation is that TAC signals through Notch/RBPJ to promote angiogenesis. Contrary to our initial hypothesis, doxycyclineactivated iNICD did not affect the CC ratio (Fig. 2a), suggesting that TAC-induced microvascularization does not involve Notch.

To probe the mechanism responsible for the increased microvascularization, we profiled the expression of mRNAs encoding 29 secreted angiogenic factors. Five were upregulated in the cKO relative to WT, including factors involved in sprouting and endothelial cell migration (for example, VEGF-A and PGF), or stabilization (THBS2), and just a single factor (TGF $\beta 3$ ) was downregulated (Fig. 2l). iNICD did not upregulate any angiogenic factor mRNAs (but downregulated Vegfa, Tnf and Ang2) despite upregulating the canonical Notch/RBPJ target genes Hes 1 and Hey2 (Fig. 1j). Although consistent with no effect of iNicd on the $\mathrm{CC}$ ratio, the result was puzzling since the angiogenic factor genes behaved differently than canonical Notch/RBPJ target genes Hes 1 and Hey2.

We investigated the divergent effects of the cKO and iNICD genotypes on angiogenic factor expression using $V e g f a$ as a paradigm. Consistent with a Notch-independent role, VEGF-A protein levels were elevated in $\mathrm{CKO}$, but not iNICD, heart tissue relative to WT controls (Fig. 2m,n; Supplementary Fig. 6). Culture media conditioned by $\mathrm{cKO}$ isolated ACM showed an increased ability to form microvascular endothelial tubes in vitro relative to WT-conditioned media, and this increased activity was blocked by the VEGF receptor inhibitor (carbozantinib) (Fig. 2o). Taken together, the in vitro and in vivo data support the model that RBPJ inhibits VEGF-A dependent angiogenesis. Chromatin 

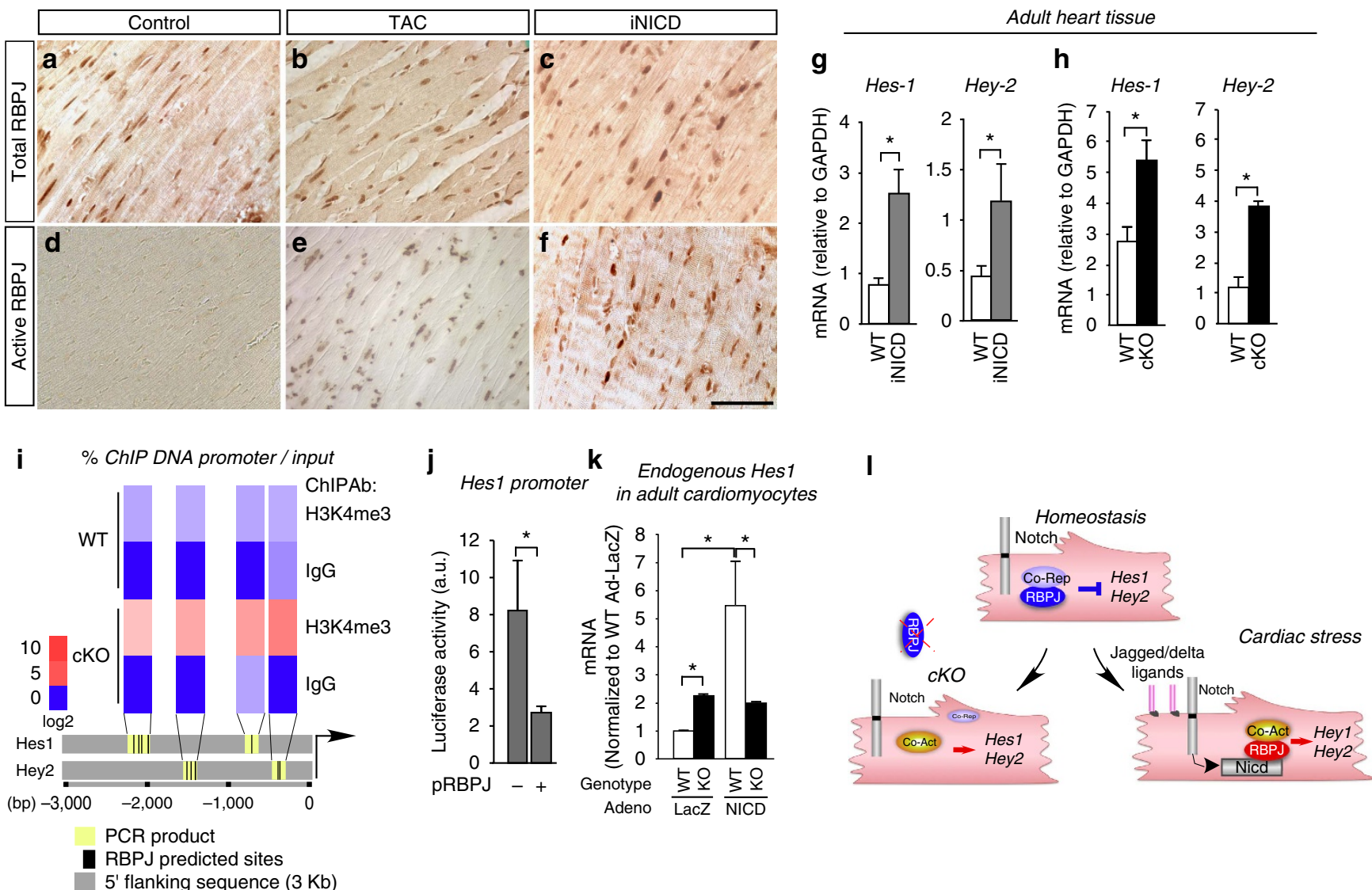

Figure 1 | Cardiac stress switches RBPJ from transcriptional repression to activation. (a-f) Histological sections of ventricular myocardium from WT mice from sham $(\mathbf{a}, \mathbf{d})$ and TAC (b,e) operated mice, and a cardiomyocyte-specific doxycycline-iNICD mouse (c,f) immunostained for total RBPJ (a-c) and RBPJ in its transcriptional activating complex(brown) (d-f). TAC and iNICD upregulate nuclear-localized RBPJ. Scale bar, $50 \mu \mathrm{m}$. (g,h) qRT-PCR of Notch target genes (Hes1, Hey2) in ventricular tissue from iNICD ( $\mathbf{g}$ ) and RBPJ CKO (h) with their respective WT controls showing upregulation of target genes in both cases. Error bars indicate SEM $n=4$ mice for each condition. mRNA levels were normalized to Gapdh levels. Asterisk, $P<0.05$. (i) Heatmap representation of ChIP analysis of $\mathrm{H} 3 \mathrm{~K} 4 \mathrm{me}^{3}$ in the endogenous Hes1 and Hey2 loci of WT and cKO ventricular myocardium. PCR primers span RBPJ consensus binding motifs (see inset below heatmap). Specific H3K4me $\mathrm{e}^{3}$ and lgG PCR product levels were normalized to input DNA (see Methods section), revealing upregulated levels (red tones) of $\mathrm{H} 3 \mathrm{~K} 4 \mathrm{me}^{3}$ modified chromatin in the cKO relative to WT. (j) Activity of transfected RBPJ on a Hes1 promoterluciferase construct in HEK 293 T cells. Error bars indicate SEM $n=5$ per condition. Asterisk, $P<0.05$. (k) Activity of endogenous Hes 1 expression ( $(\mathrm{RT} T-P C R$ ) in isolated ACM, $48 \mathrm{~h}$ after adenoviral infection to overexpress NICD compared to inert gene (LacZ) control. Error bars indicate SEM $n=4$ mice for each condition. mRNA levels were normalized to $\beta$-actin levels and plotted relative to WT Ad-LacZ condition. Asterisk, $P<0.05$. (I) Model showing that RBPJ toggles between transcriptional repression (basally) and activation in response to Notch activation. Loss of RBPJ (cKO) de-represses target genes.

immunoprecipitation revealed increased content of histone-H3 modifications (K9/K18Ac ${ }^{2}$ and $\left.\mathrm{K}_{\mathrm{m}} \mathrm{me}^{3}\right)$ in the endogenous Vegfa promoter in a region $\sim 1.5 \mathrm{~kb}$ upstream of the start site of transcription that contains both RBPJ and HIF consensus motifs (Fig. 2p; Supplementary Table 3). These epigenetic marks indicate gene activation in the cKO cardiomyocytes. Activation was reversed by the overexpression of RBPJ, and co-transfection with a truncated-form of SHARP that physically sequesters RBP ${ }^{18}$ completely eliminated repression (Fig. 2q). Thus, RBPJ represses Vegfa gene expression under unstressed conditions, and deleting RBPJ leads to Vegfa gene activation (Fig. 2r).

Cardioprotection and hypoxia tolerance in rbpj cKO hearts. Given the increased CC ratio in the cKO heart, we evaluated the hemodynamic consequences deleting Rbpj. In addition to the cKO genotype, we generated a tamoxifen-inducible Myh6-Cre ${ }^{19}$ knockout (icKO; Myh $6^{\mathrm{mER}-\mathrm{Cre}-\mathrm{mER} /+}, R b p \mathrm{f}^{\mathrm{fl/fl}}$ ) to afford precise control over the timing of Rbpj deletion. Blood flow within left ventricular myocardium was greater in the icKO and cKO hearts than in WT controls (Fig. 3a). icKO and cKO mice (along with genotype-specific WT controls) were subjected to acute hypoxia by gradually decreasing ambient $\mathrm{O}_{2}$ from 21 to $5 \%$. The different groups (cKO, icKO and their WT controls) had similar hemodynamic values under normoxic conditions $\left(21 \% \mathrm{O}_{2}\right)$ (Supplementary Table 4). At $5 \% \mathrm{O}_{2}$, vascular resistance (VR) increased as expected, but was significantly lower in $\mathrm{cKO}$ and icKO than WT mice (Fig. 3b), correlating with elevated mean arterial pressure (MAP) and cardiac output (CO) (Fig. 3c-f). Although not statistically significant, $\mathrm{O}_{2}$ delivery at baseline and $5 \% \mathrm{O}_{2}$ was greater in the $\mathrm{cKO}$ and icKO relative to WT (Fig. 3g). Myocardial hypoxia (measured on histological section) in the cKO and icKO was consistently diminished by $10-20 \%$ relative to WT controls (Fig. 3h,i). Thus, loss of RBPJ in ACM correlated with increased myocardial oxygenation and $\mathrm{CO}$ under hypoxia.

Since stimulating angiogenesis has been considered a potential therapy for ischaemic disease $e^{5,6,20}$, we evaluated the response of icKO mice to MI by serial echocardiography and histology (Fig. 3j). The icKO hearts had increased CC ratio at baseline and preserved cardiac function (Fig. 3l) and morphology (Fig. 3m) relative to WT hearts post-MI (Fig. 3l,m), here measured in the 


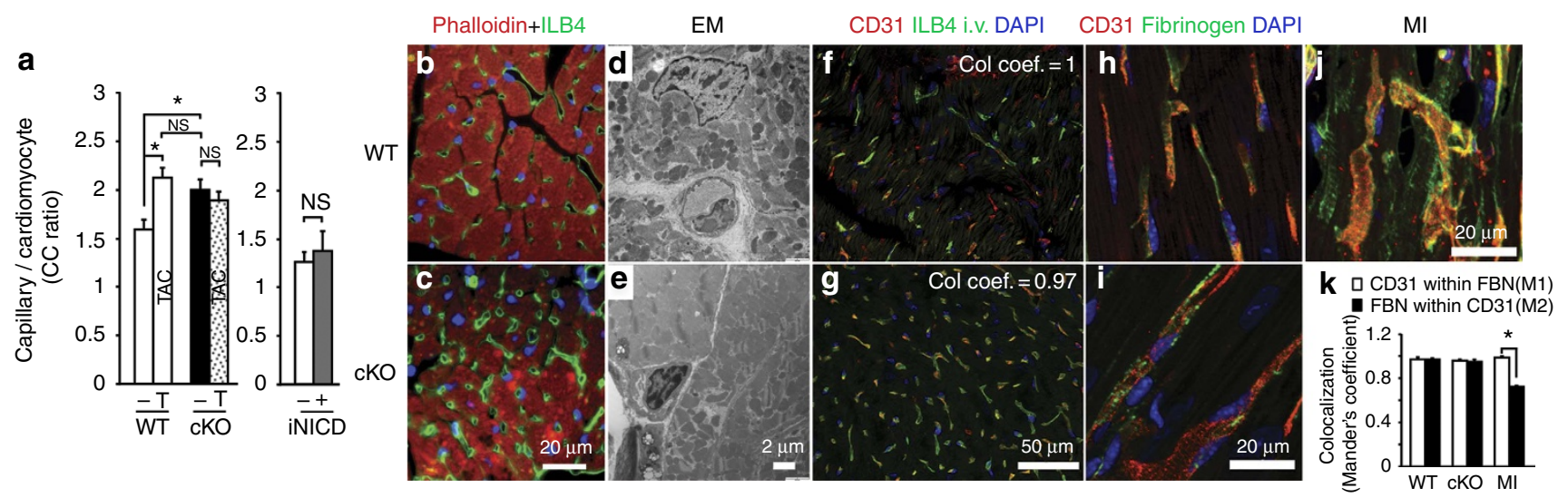

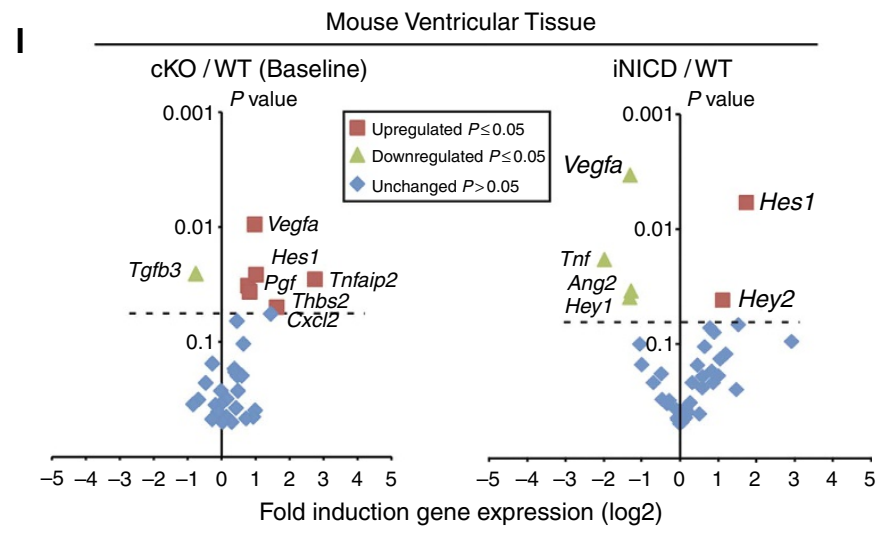

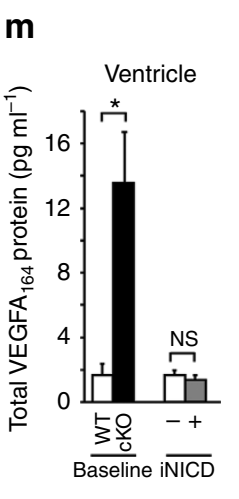

n Adult
cardiomyocytes

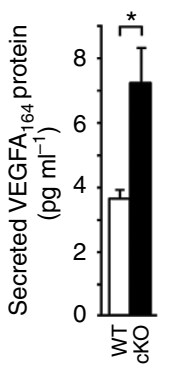

Adult cardiomyocytes-
conditioned media

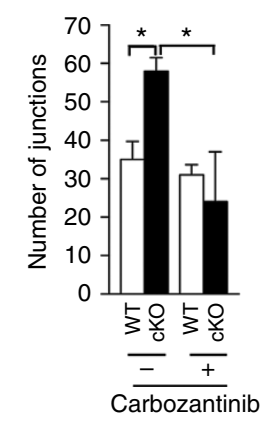

p
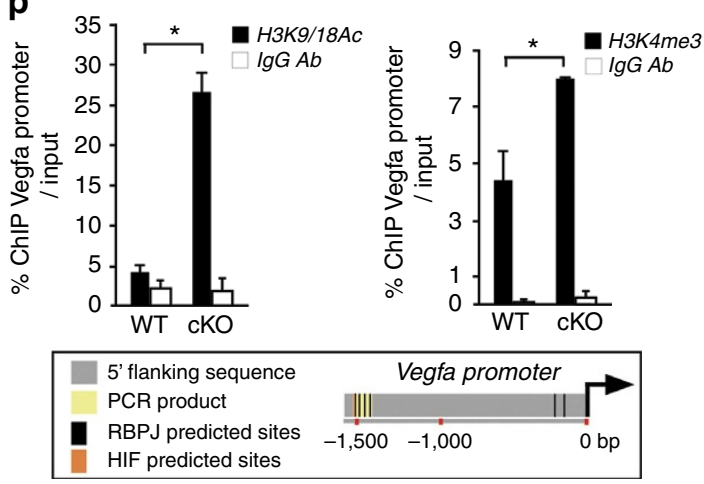

q

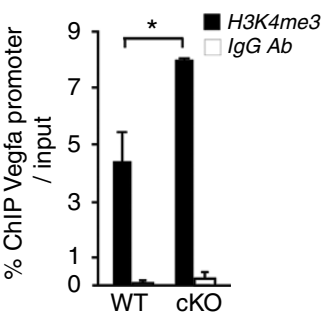

$\begin{array}{rr}\text { pRBPJ } & -++++ \\ \text { Sharp2002 } & --\end{array}$

$\mathbf{r}$

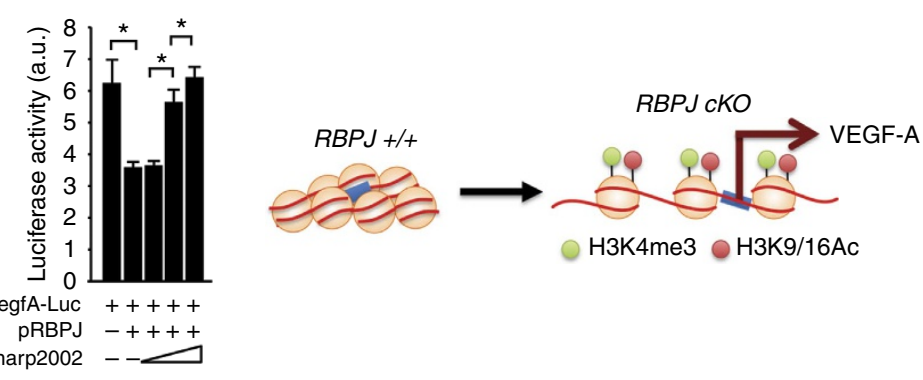

Figure 2 | Cardiomyocyte-specific knockout of RBPJ increases myocardial angiogenesis. (a-c) Microvessel density quantification (a) on histological

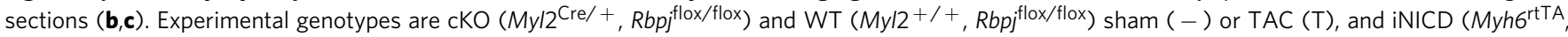
tetO-NICD) with $(+)$ or without $(-)$ doxycycline (DOX). $n=4$, mice for all conditions except WT/ +TAC were $n=3$. Scale bar, $20 \mu \mathrm{m}$. $(\mathbf{d}, \mathbf{e})$ Electron microscopy of WT (d) and cKO (e) capillaries at 48 months. Scale bar, $2 \mu \mathrm{m}(\mathbf{f}, \mathbf{g})$ Perfusion analysis by ILB4 and CD31 co-localization after FITC-ILB4 injection. $n=3 \mathrm{WT}$, and $5 \mathrm{cKO}$ mice. Scale bar, $50 \mu \mathrm{m}$. (h-k) Co-localization between fibrinogen (green) and CD31 (red) in WT (h), cKO (i) and control WT-infarcted heart (MI) reflecting capillary integrity. $(\mathbf{k}) n=3$. Scale bar, $20 \mu \mathrm{m}$. (I) Gene expression analysis of 29 secreted angiogenic factors and 3 Notch target genes in ventricles from cKO, WT and doxycycline-induced iNICD mice by qRT-PCR normalized to ActB. Volcano plots portray the cKO to WT ratio or iNICD to WT ratio relative to $P$-value. Red and green points indicate statistically significant $(P \leq 0.05, n=4)$ induction or repression of gene expression, respectively; blue points indicate no statistically significant difference. $(\mathbf{m}, \mathbf{n})$ VEGFA 165 protein quantification by ELISA in WT, cKO and iNICD heart tissue $(\mathbf{m})$ and conditioned media from WT and cKO-cultured ACM $n=4(\mathbf{n})$. (o) Conditioned media from WT and cKO ACM were applied to HMEC1 cells and network formation quantified incubation in the absence or presence of the VEGF receptor inhibitor carbozantinib $(2 \mu \mathrm{M}) . n=3$. (p) Histone marks in the endogenous Vegfa locus. ChIP with control lgG (white bars) and anti-H3K9/18Ac or anti-H3K4me (black bars) from isolated ACM was amplified using primers spanning RBPJ and HIF consensus binding motifs (schematic) and results normalized to input DNA. $n=3$. (q) Repression of Vegfa promoterluciferase (50 ng) activity by RBPJ (1 ng) and reversion by co-transfection with escalating doses (0.5, 1 and $10 \mathrm{ng}$ ) of the SHARP RBPJ-binding domain (2002-3664) (ref. 18). $n=3$. (r) Loss of RBPJ leads to acquisition of active chromatin modifications and induction of Vegfa transcription. Data are mean \pm SEM $* P<0.05$; ns, not significant; $n$, biological replicates.

interventricular septum (IVS) since the left ventricle (LV) free wall was injured by the MI. The extent of fibrotic scar formation was also diminished significantly, from $25 \pm 4.3 \%$ of the total LV area in WT to $\sim 8 \pm 3.0 \%$ in icKO hearts (Fig. $3 \mathrm{n}$ ). The area of the myocardium that was hypo-perfused acutely $(24 \mathrm{~h})$ post-MI was identical in both genotypes, quantified by FITC-ILB4 perfusion ( $\sim 25 \%$ of total LV area) (Fig. 3o), and there was no effect on arterial arborization (Supplementary Fig. 4,k,k'), 

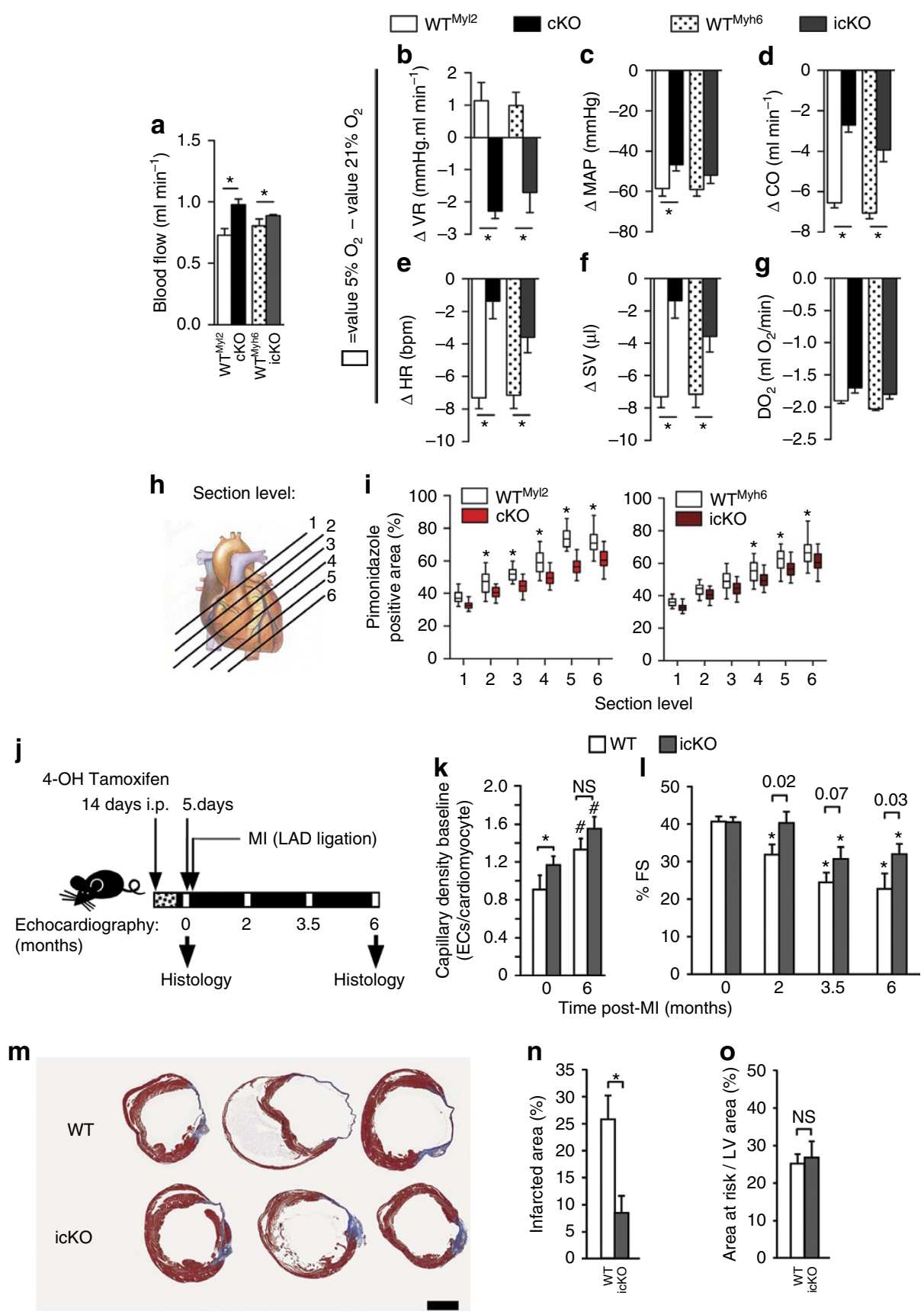

Figure 3 | Loss of RBPJ induces hypoxia tolerance and cardioprotection after MI. (a-g) Hemodynamic parameters. Blood flow at baseline (a) and hemodynamics upon hypoxemic challenge $(\mathbf{b}-\mathbf{g})$, plotted as value at 5 versus $21 \%$ inhaled $\mathrm{O}_{2}$ in cKO and icKO $\left(\right.$ Myh $\left.6^{\mathrm{mER}} \mathrm{Cre}-\mathrm{mER} /+, R b p \mathrm{j}^{\mathrm{fl} / \mathrm{fl}}\right)$ mouse lines relative to their respective genotype controls (WTMyl2 or WTMyh6). Parameters were VR (b), MAP (c), CO (d), HR (e), SV (f), and $\mathrm{O}_{2}$ delivery $\left(\mathrm{DO} \mathrm{C}_{2}\right)$ $(\mathbf{g}) . n=4 ;{ }^{\star} P<0.05$ (ANOVA). (h,i) Tissue hypoxia quantified by pimonidazole detection in parallel histological sections, diagrammed (h). Apical sections (towards 6 ) had significantly smaller hypoxic regions in the cKO (left graph) and icKO (lower graph) hearts compared to their WT controls (j). Box represents mean and 25th and 75th percentiles; whiskers 5th and 95th percentiles. $n=4$ mice (j) Experimental protocol for $\mathrm{MI}$. 4-OH tamoxifen was injected to induce recombination of the icKO at daily intervals during a 14-day window ending 5 days prior to LAD ligation (MI), followed by serial echocardiography and histological analysis at endpoint. (k) Microvessel density quantified 6 months after MI on histological sections. Capillary density was examined in the IVS, rather than the anterior wall, since the IVS was not damaged by the infarction. $n=3,4,6,6$ for $\mathrm{WT}$, icKO, WT + MI, icKO + MI, respectively. (I) LV fractional shortening (\%FS) ( $n=9$ for each genotype and timepoint). $P$ value between genotypes listed. ${ }^{\star} P<0.05$ respect to baseline same genotype. $(\mathbf{m}, \mathbf{n})$ Cross-sectional views $(\mathbf{m})$ of WT and icKO hearts 6 months after MI and stained with Masson's trichrome at the level of the papillary muscles. Quantification of the scar area (n) in infarcted myocardium (blue histological stain) expressed as a fraction of LV circumference from 3 parallel cross-sections spaced $700 \mu \mathrm{m}$ apart ( $n=6 \mathrm{WT}$ and 7 icKO; see Methods section). Scale bar, $1 \mathrm{~mm}$. (o) LV hypo-perfusion (Area At Risk, AAR) determined by intravenous injection of FITC-ILB4 2-days post-MI and quantified on histological sections at the level of papillary muscles $(n=3$ mice each genotype, see Methods section). Note that both genotypes showed comparably sized areas of hypo-perfusion. Data are means \pm SEM. ${ }^{\star} P<0.05 ;$ ns, not significant; $n$, biological replicates. 
demonstrating that the immediate effect of left anterior descending (LAD) occlusion was the same in both genotypes. We conclude that deleting RBPJ improved tolerance to MI.

RBPJ physically inhibits HIFs. The preceding showed that deleting RBPJ increases angiogenic factor gene expression and microvessel density, correlating with improved cardiac function after MI. We then investigated the mechanism responsible for the puzzling finding that RBPJ represses angiogenic factor genes basally, but that Notch/RBPJ does not induce them. Since hypoxia is a major regulator of angiogenesis ${ }^{2,6,21}$, we examined whether hypoxia responsiveness is altered by the cKO. Exposing isolated ACM to severe hypoxia $\left(1 \% \mathrm{O}_{2}\right)$ upregulated 13 and downregulated 4 of the 29 secreted angiogenic factor genes profiled relative to normoxia $\left(21 \% \mathrm{O}_{2}\right)$ (Supplementary Fig. 7). The response at $1 \% \mathrm{O}_{2}$ was nearly identical in WT and cKO genotypes. However, plotting the ratio of gene expression change in $\mathrm{cKO}$ relative to WT revealed that the loss of RBPJ sensitized angiogenic genes to induction by moderate hypoxia $\left(6-3 \% \mathrm{O}_{2}\right)$ (Fig. 4a).

Given that the $-1.5 \mathrm{~kb}$ region of the Vegfa promoter contains both RBPJ and HIF consensus binding sites (Fig. 4b, inset; Supplementary Table 3), we hypothesized that RBPJ might interact with HIF to mediate repression. In a luciferase assay, RBPJ repressed induction of the Vegfa promoter by hypoxia (Fig. 4b) as well as by HIF1 $\alpha$ or Hif $2 \alpha$ (Fig. 4c) in a dosedependent manner.

Repression occurred identically with a mutant RBPJ containing four mutations that block binding to recognition sites in $\mathrm{DNA}^{22}$ (Fig. 4d), suggesting that RBPJ does not need to interact with DNA in order to inhibit HIF.

Overexpressed GFP-tagged RBPJ in HEK293T cells under hypoxia was co-immunoprecipitated with endogenous HIF $1 \alpha$ and HIF $2 \alpha$ (Fig. 4e), suggesting a physical interaction between the proteins. Furthermore, a proximity ligation assay (PLA, Fig. 4f) revealed that RBPJ co-localizes with both HIF $1 \alpha$ and HIF $2 \alpha$ in ACM under normal and hypoxic conditions in vitro. Cytoplasmic and nuclear co-localization was apparent, consistent with the distribution of HIF and RBPJ in both compartments (Supplementary Fig. 8) and the ability of the RBPJ DNA-binding mutant to antagonize hypoxia and HIF-dependent Vegfa transcription. To control for non-specific interactions, no fluorescent signal was detected in cKO cardiomyocytes (Fig. 4f). Both HIF $1 \alpha$ and HIF $2 \alpha$ are detectable in WT and cKO mouse hearts (Fig. 4g), although HIF $2 \alpha$ is more prominent in nuclei $(65 \%(\mathrm{HIF} 2 \alpha)$ versus $15 \%(\mathrm{HIF} 1 \alpha)$ in WT cells) and increases further in the cKO $(80 \%$ (HIF $2 \alpha)$ versus $15 \%$ (HIF $1 \alpha)$ in cKO cells) (Fig. 4h; Supplementary Fig. 9). These data indicate that RBPJ physically interacts with HIF proteins basally, and that repression is overcome by HIF stabilization under hypoxia.

\section{Discussion}

In summary, $\mathrm{RBPJ}$ regulates a paracrine circuit for myocardial angiogenesis (Fig. 4i). RBPJ exerts control over microvessel density by acting within cardiomyocytes to antagonize HIF $1 \alpha$ and HIF $2 \alpha$; therefore, desensitizing the angiogenic response to hypoxia. Deleting RBPJ, therefore, increases sensitivity to hypoxia. Attenuating RBPJ in cardiomyocytes is remarkable, in that it benignly increases myocardial vascularization, increases cardiac perfusion and output under hypoxia and preserves cardiac function following MI. There are relatively few instances of RBPJ having a repressive role in mammals ${ }^{23-25}$, and most studies have focused primarily on its function as the effector of Notch signalling, which is itself cardioprotective following ischaemic injury $8,9,26$. The effect of RBPJ deletion resembles chronic adaptation of the heart to hypoxia ${ }^{27}$ suggesting that attenuating the suppressive function of RBPJ might be therapeutic in the setting of ischaemic injury or chronic overload.

\section{Methods}

All animal handling and care followed the NIH Guide for Care and Use of Laboratory Animals. The experimental protocols were approved by Institutional Animal Care and Use Committees of either the Sanford-Burnham-Prebys Medical Discovery Institute, the University of California San Diego or Washington University. Unless otherwise noted, approximately equal numbers of 8-16-weekold male and female mice were used in all studies.

Genetic ablation of RBPJ in adult mouse hearts. To obtain cardiomyocytespecific deletion of RBPJ in adult mouse hearts we created the following mouse lines. $M y l 2^{\mathrm{Cre} /+}$ transgenic mice (Black Swiss) were crossed to $R b p j^{\text {flox } / \text { flox }}$ mice $(\mathrm{C} 57 \mathrm{BL} / 6)^{28}$ to yield cKO $\left(M y l 2^{\mathrm{Cre} /+}, R b p j^{\text {flox/flox }}\right)$ and phenotypically WT $\left(M y l 2^{+l+}, R b p j^{\text {flox/flox }}\right)$ littermates. $M y h 6^{\text {cre/Esrl }}$ transgenic mice ${ }^{19}$ were also crossed to $R b p j^{\text {flox/flox }}$ mice to obtain cKO Myh $6^{\mathrm{mER}-\mathrm{Cre}-\mathrm{mER} /+}, R b p j^{\mathrm{fl} / \mathrm{fl}}$ and phenotypically WT $M y h 6^{\text {mER-Cre-mER/+ }}, R b p j^{+1+}$

Tamoxifen induction of recombination. 4-OH tamoxifen (Sigma) prepared as $5 \mathrm{mg} \mathrm{ml}^{-1}$ in corn oil was injected $(0.1 \mathrm{ml}$ per mouse) intraperitoneally at daily intervals during a 14-day period that ended 5 days before LAD ligation, as described $^{29}$, or 30 days before physiology tests.

Genetic activation of Notch receptor in adult mouse hearts. $\alpha M H C r t T A$; tetO-NICD mouse line was previously generated ${ }^{15}$ to activate Notch signalling by inducing NICD specifically in ACM. NICD was induced with doxycycline chow (BioServ $200 \mathrm{mg} \mathrm{kg}^{-1}$ ) during 2 weeks. $\alpha M H C r t T A$ and tetO-NICD littermates fed doxycycline were used for comparison in all conditional gene expression experiments unless otherwise noted. Littermate controls were used for all experiments. Animal protocols involving these mice were approved by the Animal Studies Committee at Washington University.

Statistical analysis. Data are shown as means \pm standard error of the mean (SEM) unless otherwise specified. $P$ values were calculated by the Mann-Whitney, the Student's t-test or the one-way ANOVA, for samples $n=3$ and $n \geq 4$, respectively, from independent biological replicates and the two-way ANOVA for physiology experiments.

Heatmap analysis. Quantitative reverse transcription polymerase chain reaction (qRT-PCR) data was internally normalized for each genotype and condition to $A c t B$ levels. The amount of complimentery DNA (cDNA) used was titrated and verified to yield cycle threshold (CT) values in the linear response range. All conditions where normalized to the values of WT cells at $21 \%$ oxygen levels. Resulting data was hierarchically clustered using Cluster 3.0 using the complete linkage cluster method. Java TreeView software generated the colour map.

Inmunocytochemistry and histochemistry. Hearts were arrested in diastole by perfusion with $300 \mathrm{mM} \mathrm{KCl}$ solution before fixation in $4 \%$ paraformaldehyde in PBS. They were then processed for either paraffin embedding or fresh frozen directly in optimum cutting temperature compound (OCT) directly after collection. Briefly, paraffin sections ( $7 \mu \mathrm{m}$ thick) were de-paraffinized, rehydrated and heat-treated in Degloacker buffer $\mathrm{pH} 9$ for antigen retrieval. After several PBS washes, sections were blocked with 5\% BSA in PBS and incubated overnight at $4{ }^{\circ} \mathrm{C}$ with the rat primary anti-RBPJ antibody (generously provided by Professor $\mathrm{T}$. Honjo, Kyoto University ${ }^{30}$ ) (1:50), rabbit monoclonal RBPJ (CST) (1:300 or $1: 2,000)$, rabbit anti-Jagged-1 (SCBT) $(1: 300)$, rabbit anti-Delta-Like 4 (SCBT) (1:100), mouse $\alpha$-actinin (Sigma) (1:500), rabbit HIF1alpha (1:100 or $1: 1,000$ for TSA) and HIF2 alpha (1:100 or 1:1,000 for TSA) (Nobus). After PBS washing, sections were incubated with secondary antibodies. Either an HRP-conjugated antirat IgG, HRP-conjugated anti-rabbit IgG, alexa fluor-conjugated (488 or 586) goat anti-mouse or goat anti-rabbit IgG were applied for one hour at room temperature. HRP was detected by DAB or by the thyramide signal amplification system (Molecular Probes) for Hif1 and HIF2 alpha. To visualize microvessels, sections were incubated with FITC-conjugated Lycopersicon esculentum agglutinin (FITCLEA, Sigma) $(1: 1,000)$ and Rhodamine-phalloidin (Invitrogen) $(1: 1,000)$ for $2 \mathrm{~h}$ in PBS with calcium and magnesium, and subsequently counterstained with DAPI. Sections were mounted and photographed on a Zeiss Axiophot microscope with a $40 \times$ objective (N.A. 0.75 ) or on a Zeiss Imager.Z1 plus apotome with $40 \times($ N.A. $1.2 \mathrm{w})$ objectives.

Hif1 and HIF2 alpha antibodies were validated on heart tissue from embryonic day E11.5 mouse where Hif1 and HIF2 alpha had been deleted. The genotype of the HIF1 and HIF2 conditional KO mice correspond to FGata5 Cre $+\times$ Hifla F/ and FGata5 Cre/ $+\times$ Hif2a F/ - , respectively. The FGata5 Cre mice have direct Cre recombination in the epicardium as well as in regions of the myocardium ${ }^{31}$. 

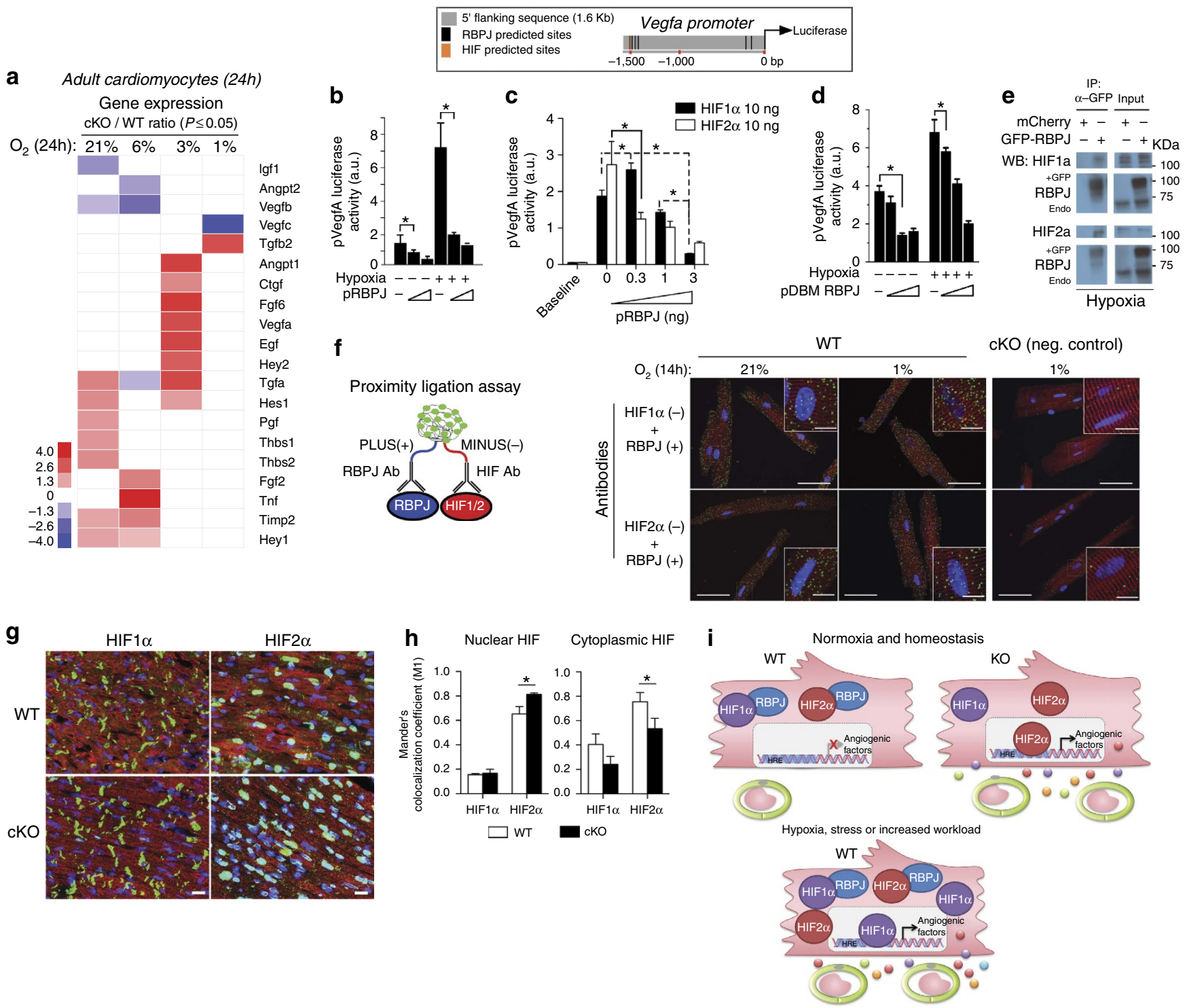

Figure 4 | RBPJ suppresses myocardial angiogenesis by antagonizing HIFs. (a) The heatmap represents the ratio of cKO to WT gene expression for statistically significantly induced (red) or repressed (blue) genes (Mann-Whitney, $n=4, P \leq 0.05$; see Methods section) in isolated cardiomyocytes cultured for $24 \mathrm{~h}$ at the indicated $\mathrm{O}_{2}$ levels. White indicates no differences. Inset to left shows colour scale. See Supplementary Fig. 7 for complete dataset. (b-d) Vegfa promoter (see inset) driving luciferase (50 ng) was co-transfected with increasing amounts (1-5 ng) of an RBPJ expression plasmid (pRBPJ) and is activity tested after $24 \mathrm{~h}$ at baseline, hypoxia $\left(1 \% \mathrm{O}_{2}\right)(\mathbf{b})$ or upon activation by HIF1 $\alpha$ or HIF2 $\alpha$ expression plasmids (10 ng) (c) in HEK $293 \mathrm{~T}$ cells. Increasing concentrations of the DNA-binding mutant (DBM) of RBPJ, pDBM-RBPJ also inhibited Vegfa promoter basally and as induced by $24 \mathrm{~h}$ of hypoxia $\left(1 \% \mathrm{O}_{2}\right)(\mathbf{d}) . n=3$ on (b) and 5 on (c,d) biological replicates. (e) Co-IP of eGFP-tagged RBPJ with endogenous HIF- $1 \alpha$ and HIF- $2 \alpha$ under hypoxic ( $2 \%$ O ${ }_{2}$ ) conditions. (f) Visualization of RBPJ interaction with HIF- $1 \alpha$ and HIF- $2 \alpha$ by in situ proximity ligation assay (PLA). Isolated ACM cultured for $14 \mathrm{~h}$ under hypoxia $\left(1 \% \mathrm{O}_{2}\right)$ or normoxia $\left(21 \% \mathrm{O}_{2}\right)$ were incubated with RBPJ probe (conjugated with PLUS oligonucleotide) and HIF1 $\alpha$ or HIF2 $\alpha$ probes (conjugated with MINUS oligonucleotide). Positive interactions in WT cardiomyocytes (green fluorescence) are seen in the cytoplasm $\left(\alpha-\right.$ actinin ${ }^{+}$, red) and nuclei (DAPI, blue). cKO ACM were used as a negative control to show PLA signal specificity. Pictures are representative of at least three experiments. Scale bars, $50 \mu \mathrm{m}$, insets $10 \mu \mathrm{m}$. (g,h) HIF-1 $\alpha$ and HIF- $2 \alpha$ detected (both in green) in cKO and WT heart sections, co-stained with $\alpha$-actinin (red) and DAPI (blue) (g). Manders co-localization coefficient M1 for HIF1 $\alpha$ or HIF2 $\alpha$ within nuclei (DAPI) (left graph, $\mathbf{h}$ ) or cytoplasm ( $\alpha$-actinin ${ }^{+}$). For M2 of DAPI and $\alpha$-actinin within HIF1 $\alpha$ and HIF2 $\alpha$, see Supplementary Fig. 9). $n=3$ and 4 hearts for WT and cKO mice. Scale bar, $20 \mu \mathrm{m}$. (i) Paracrine regulation of myocardial angiogenesis. RBPJ antagonizes HIFs to homeostatically repress angiogenesis. HIF stabilization in response to decreased $\mathrm{O}_{2}$ increases angiogenesis. All data are means \pm SEM. ${ }^{\star} P<0.05 ; n s$, not significant; $n$, biological replicates.

For frozen sections, tissue was fresh frozen in OCT. Histological sections were fixed in methanol/acetone $(1: 1)$ at $-20^{\circ} \mathrm{C}$ for $2 \mathrm{~min}$ and processed as described above, but the antigen retrieval step was omitted. Frozen sections were incubated with goat anti-fibrin/fibrinogen (GAM/Fbg/Bio; Nordic Immunology) (1:500), rat anti-mouse CD31 (BD-Pharmingen) (1:100), anti-alpha smooth muscle actin (Sigma) (1:500) primary antibodies and alexa fluor-conjugated (488 or 586) streptavidin or goat anti-rat or goat anti-mouse IgG (all 1:500). Biotin blocking was performed as described for the Streptavidin/Biotin blocking kit (Vector Labs) on those sections incubated with biotin-conjugated antibodies.
For histochemistry, hearts were fixed in $4 \%$ formaldehyde and processed for paraffin embedding. Sections were stained for Massons' trichrome (collagen stain), PAS (periodic acid acid-Schiff) (to detect glycogen) and Oil red-O (to detect lipid) following standard procedures (Sanford-Burnham-Prebys Histology Core Facility). Sections were mounted and photographed on a TE300 Nikon microscope with $40 \times$ (N.A. 0.75 ) objective.

For plastic casting of coronary vascular trees, Microfil polymer (Flow Tech, Inc.) was perfused through the aorta of isolated hearts ${ }^{2}$. Mice had been previously injected with heparin and the heart washed with saline solution to remove blood. 
After polymer injection hearts were processed following manufacturer's instructions and imaged on a Nikon SMZ stereo-microscope.

Electron microscopy. For transmitted electron microscopy, hearts were arrested in diastole by perfusion with $300 \mathrm{mM} \mathrm{KCl}$, dissected and sliced in $1 \mathrm{~mm}$ thick sections and fixed in $2 \% \mathrm{pFA} / 2 \%$ glutaraldehyde in $0.1 \mathrm{M}$ cacodylate buffer solution, $\mathrm{pH}$ 7.4. Slices were then washed and incubated in $1 \%$ osmium tetroxide in the same buffer, dehydrated in ethanol and embedded in Eponate 12 epoxy resin (Ted Pella Inc. C, Redding, CA, USA). Ultrathin sections were prepared on a Reichert-Jung Ultracut microtome and stained with uranyl acetate and lead citrate, and examined using a Hitachi $\mathrm{H}-600 \mathrm{~A}$ electron microscope, at $75 \mathrm{kV}$. Digital images were acquired with $2 \mathrm{~K} \times 2 \mathrm{~K}$ CCD cooled monochrome 12 -bit camera L3C (SIA, Atlanta, GA, USA) using "Picture Frame" software (Optronics, Inc., Goleta, CA, USA). Images were processed with ImageJ (public domain software) to achieve a similarity in contrast and brightness of all images.

Imaging quantification. Imaging quantification was done with ImageJ. Colocalization analysis was performed using a colocalization analysis plugin (http://www.uhnres.utoronto.ca/facilities/wcif/imagej/colour_analysis.htm) that generates Mander's coefficients ${ }^{32}$. Determination of distances on images (for example, Z-line and cardiomyocyte cross-sectional area measurements) were done by manually selecting the distances to be measured. For the analysis of the size of ACM in vitro, brightfield images were process on ImageJ as described (http://rsbweb.nih.gov/ij/docs/pdfs/examples.pdf) and quantified using the particle size analysis function.

Intravital endothelial cell labelling. Mice were anesthesized by intraperitoneally (i.p.) ketamine $\left(100 \mathrm{mg} \mathrm{kg}^{-1}\right)$-xylazine $\left(2.5 \mathrm{mg} \mathrm{kg}^{-1}\right)$ injection followed by injection with heparin $\left(1,000 \mathrm{U} \mathrm{kg}^{-1}\right)$ to reduce formation of thrombi. To label the endothelial cells intravitally, the anesthetized mice received a jugular injection of $0.1 \mathrm{ml}$ FITC-conjugated Isolectin B4 (ILB4, Vector Lab). ILB4 was allowed to circulate for $15 \mathrm{~min}$. Mice were then sacrificed and hearts collected in $4 \%$ paraformaldehyde, washed in PBS, cryopreserved in sucrose, and embedded in OCT and process for frozen sections.

ACM preparation and culture. ACM were isolated from 3 to5-month-old mice by Langendorff perfusion method as described ${ }^{33}$. Before plating, cardiomyocytes were separated from non-cardiomyocytes by sedimentation. Two hours after plating, adenovirus was applied to the media at an MOI of $\sim 50$ pfu per cell for $24 \mathrm{~h}$. After changing media on the next day, cardiomyocytes were incubated in a hypoxia chamber (Biospherix XVivo, UK) under specified $\mathrm{O}_{2}$ tension, as indicated in the text, for $24 \mathrm{~h}$ before collection of samples for gene expression analyses or immunodetection.

Co-immunoprecipitation and western blot. For HIF1a and HIF2a interaction with RBPJ, HEK 293 T (ATCC) cells were transfected with either mCherry (negative control) or RBPJ-GFP expression vectors and subjected to hypoxia $\left(2 \% \mathrm{O}_{2}\right)$ overnight before total protein extraction. Total extracts were prepared by incubating cells at $4{ }^{\circ} \mathrm{C}$ in lysis buffer (PBS with $0.5 \%$ Triton-X 100). Lysates were centrifuged for $1 \mathrm{~min}$ at $12,000 \mathrm{~g}$ to remove insoluble debris. Immunoprecipitation was preformed by incubating total extracts with anti-GFP antibody (1:100) preadsorbed to protein A/G-agarose (Santa Cruz Biotechnology) and rocking for $1 \mathrm{~h}$ at $4 \mathrm{C}$. Beads were then washed $3 \times$ with $1 \mathrm{ml}$ of ice-cold lysis buffer prior to resuspension in $1 \times$ Laemli buffer and subjected to western blot. Antibodies used for western blot were RBPSUH (D10A4) XP (Cell Signaling) (1:1,000), HIF-1 alpha (NB100-479) (1:500) and HIF-2 alpha (NB100-122) (1:500).

In situ PLA and immunofluorescence. Isolated ACM seeded on coverslips were fixed on cold methanol for $10 \mathrm{~min}$. After washing with PBS and permeabilization with $1 \%$ Triton X-100 cells were process for PLA or immunofluorescence.

For immunofluorescence, cardiomyocytes were incubated with blocking buffer (5\% BSA, $0.1 \%$ Triton X-100) alone for $1 \mathrm{~h}$ and then with the primary antibodies overnight at $4{ }^{\circ} \mathrm{C}$. Excess of antibody was removed by several PBS washes and alexa fluor-conjugated (488) secondary antibody with rhodamine-conjugated phalloidin $(1: 1,000)$ was applied with the blocking buffer for $1 \mathrm{~h}$. After several PBS washes, coverslips were mounted on slides with Vectashield with DAPI (Vector Labs) and photographed.

The proximity ligation assay was performed on coverslips following the Duolink In Situ User Manual (sigma.com/duolink) and using Duolink In Situ Solutions (Sigma). First, we made PLA probes conjugating primary antibodies with a PLUS or MINUS oligonucleotide. We used the Duolink In Situ Probemaker PLUS (Sigma) for RBPJ antibody, RBPSUH (D10A4) XP (Cell Signaling) and MINUS for HIF-1 alpha (NB100-479) and HIF-2 alpha (NB100-122) antibodies. After blocking, cardiomyocytes were incubated with combinations of HIF-1 alpha probe $(-)$ and RBPJ probe $(+)$, or HIF-2 alpha probe $(-)$ and RBPJ probe $(+)$ overnight at $4{ }^{\circ} \mathrm{C}$ in a humidified chamber (all 1:250). After washing the antibody, we performed the ligation and amplification steps as described (sigma.com/ duolink) using the Duolink In Situ Detecton Reagents Green (Sigma). After the final washes we incubated cells with rodamine-conjugated phalloiding for $10 \mathrm{~min}$ before mounting the coverslips on glass slides using Vectashield with DAPI (Vector Labs).

Images were taken on a Zeiss Imager.Z1 plus apotome with $40 \times($ N.A. $1.2 \mathrm{w})$ objectives and processed with ImageJ (NIH, Rasband, W.S., ImageJ, U. S. National Institutes of Health, Bethesda, Maryland, USA, http://imagej.nih.gov/ij/, 1997-2014).

Protein analysis. Mouse VEGF-A ELISA (Biosource, Invitrogen) was performed following manufacturer's instructions. ACM were isolated as described (see above) from WT sham and TAC mice and immediately collected on RIPA lysis buffer. Western blots were performed as described ${ }^{34}$. Briefly, samples were loaded and run on a $4-12 \%$ TGX-precast gels (Bio Rad) after being heated on $2 \times$ Laemmli sample buffer with $\beta$-mercaptoethanol (Bio $\mathrm{Rad}$ ) for $10 \mathrm{~min}$ at $70^{\circ} \mathrm{C}$. proteins were then transferred using the Trans-Blot Turbo Transfer System (Bio Rad) into PVDF membranes that were blocked with $5 \%$ Difco skin milk (BD Bioscience) before antibody incubations. Antibodies used were rat primary anti-RBPJ (1:500) antibody (generously provided by Professor T. Honjo, Kyoto University ${ }^{30}$ ), ANF (1:500) (Millipore, AB5490) and GAPDH (1:2,000) (Abcam, ab9485).

Endothelial tube formation assay. A microvascular endothelial tube formation assay was performed as described ${ }^{35}$ to quantify microvessel formation in response to factors produced by the WT and cKO cardiomyocytes. Briefly, media conditioned by $18 \mathrm{~h}$ culture of mouse ACM was collected and centrifuged to remove dead cells and debris. The conditioned media was then applied to human microvascular endothelial (HMEC1) cells plated in 96-well plates previously coated with matrigel $\left(7-8 \mathrm{mg} \mathrm{ml}^{-1}, 45 \mu \mathrm{l}_{\text {well }}{ }^{-1}\right)$. The cultures were incubated for an additional $16 \mathrm{~h}$ either with or without the VEGF receptor inhibitor carbozantinib (Selleckchem). After the $16 \mathrm{~h}$ culture period, the angiogenic response was quantified automatically by determining the number of junctions formed using the macro 'Angiogenesis Analyzer' by Gilles Carpentier (Gilles Carpentier. Contribution: Angiogenesis Analyzer, ImageJ News, 5 October 2012) for NIH Image J 1.47v Program.

RNA extraction and qRT-PCR. RNA was extracted from cells and tissue using Trizol reagent (Invitrogen) and cDNA was made using the Qiagen Reverse Transcription kit. PCR with specific primers (see Supplementary Tables 5 and 6) was performed using the LightCycler FastStart Master SYBR Green Kit (Roche) in a LightCycler 2.0 (Roche). Gapdh and $\beta$-actin (Actb) were used as internal controls to normalize the data, as specified in the text.

For the angiogenic gene analysis, quantitative Real-Time PCR was performed for the different samples using the iQ SYBR Green Supermix (BioRad) and loaded on a ABI $7900 \mathrm{HT}$. Values are expressed either as $2^{\Delta \Delta \mathrm{Ct}}$, with $\Delta \Delta \mathrm{Ct}$ defined as the difference in crossing threshold $(\mathrm{Ct})$ values between experimental and control samples as described ${ }^{36}$ or as $\log _{10} 2^{\Delta \mathrm{Ct}}$ using $A c t b$ ( $\beta$-actin) as control gene.

Luciferase assay. Luciferase reporter constructs were transfected into HEK293T cells on 24 or 384 well plates with Lipofectamine LTX. Overall, $24 \mathrm{~h}$ after transfection, cells were placed under hypoxia for $12-20 \mathrm{~h}$ as above. Samples were processed to assay luciferase activity using the Dual Luciferase Kit (Promega). Luciferase activity was normalized against total protein content quantified by Bradford colorimetric analysis (Pierce). pHes1 was a gift from Jan Jensen (Cleveland Clinic, Ohio); mouse $1.6 \mathrm{~Kb}$ pVegfa-Luc from Patricia D'Amore ${ }^{37}$ (Harvard Medical School, Boston, MA, USA), pSharp (2002-3664) DeltaB and pRbpj from Franz Oswald (Max-Planck-Institute of Immunobiology, Germany), HA-HIF1alpha-pcDNA3 and HA-HIF2alpha-pcDNA3 was a gift from William Kaelin (Addgene plasmid \# 18949 and Addgene plasmid \# 18950, respectively) ${ }^{38}$

Chromatin immunoprecipitation (ChIP) analysis. ChIP assays were performed using previously described methods ${ }^{39}$. Briefly, ACM from WT and RBPJ cKO mouse hearts were collected, fixed in $2 \%$ formaldehyde $12 \mathrm{~min}$ at room temperature, rinsed twice in ice-cold PBS, and lysed directly in sonication buffer ( $1 \%$ SDS, $10 \mathrm{mM}$ EDTA, $50 \mathrm{mM}$ Tris- $\mathrm{HCl} \mathrm{pH} 8.0$ and protease inhibitors cocktail (Sigma)). The resulting cell suspension was sonicated in a waterbath sonicator (MiSonix Sonicator 3000) using the following programme: $30 \mathrm{~s} \mathrm{ON,} 30 \mathrm{~s}$ OFF during10 min (Output Power: 4). The suspension was centrifuged (Eppendorff microfuge) and the cleared supernatant was incubated at $4{ }^{\circ} \mathrm{C}$ overnight with an antibody to di-acetylated Histone3 (K9, K18) (Millipore, 07-593) or to tri-methylated Histone3 (K4) (Abcam Ab8580) (both $1 \mu \mathrm{g} / 10^{6}$ cells). Normal rabbit IgG was the negative control $\left(1 \mu \mathrm{g} / 10^{6}\right.$ cells). Protein-DNA complexes were collected on Protein G beads (SIGMA). After rinsing in PBS, the final DNA samples were subjected to PCR. Primer sets specific for the mouse Vegfa, proximal promoters spanning Hif and RBPJ-binding sites were synthesized for this purpose (Supplementary Table 3). PCR conditions were $95^{\circ} \mathrm{C}$ for $2 \mathrm{~min}$, followed by $25-30$ cycles at $95^{\circ} \mathrm{C}$ for $30 \mathrm{~s}, 55-60^{\circ} \mathrm{C}$ for $30 \mathrm{~s}$ and $72^{\circ} \mathrm{C}$ for $30 \mathrm{~s}$.

Transverse aortic constriction (TAC). Mice between 10 and 14 weeks of age were anesthetized with a ketamine $\left(100 \mathrm{mg} \mathrm{kg}^{-1}\right)$-xylazine $\left(2.5 \mathrm{mg} \mathrm{kg}^{-1}\right)$ mixture 
administered i.p., and connected to a rodent ventilator after tracheal intubation $^{40,41}$. The chest cavity was opened with scissors by a small incision at the level of the second intercostal space. After isolation of the aortic arch, a 7-0 silk suture was placed around the aorta and a 27 -gauge needle. The needle was immediately removed to produce an aorta with a stenotic lumen. The chest cavity was then closed with one 6-0 nylon suture and all layers of muscle and skin closed with 6-0 continuous absorbable and nylon sutures, respectively. Sham-treated animals underwent surgery without the final tightening of the constrictive suture ${ }^{40,41}$. Gradients across the stenotic aorta were measured through echocardiographic analysis, and animals with pressure gradients less than $30 \mathrm{mmHg}$ were not included in the study. Echocardiography was as described ${ }^{42}$. Both males and females were used and no significant gender difference was noted.

Hearts were collected at 14 days after the surgery, arrested in $300 \mathrm{mM} \mathrm{KCl}$ and fixed in $4 \%$ PFA, or fresh frozen in OCT for histological analysis or, frozen, and stored at $-80^{\circ} \mathrm{C}$ for RNA and protein extraction.

MI mouse model. The protocol was essentially as described ${ }^{43}$. Briefly, an oblique 8 -mm incision was made $2 \mathrm{~mm}$ away from the left sternal border toward the left armpit (1-2 mm below). The muscles were separated with care to avoid damaging blood vessels. The chest cavity was then opened at the 4 th intercostal space taking care not to damage the lung. A chest retractor was inserted and opened gently to spread the wound to $8-10 \mathrm{~mm}$ in width. The pericardium was gently picked up with curved and straight forceps, pulled apart, and placed behind the arms of the retractor. The LAD coronary artery runs in the midst of the heart wall from underneath of the left atrium toward the apex and was typically visible by its pulsating bright red colour. In instances when the LAD artery could not be visualized, the left atrium was lifted so that the source of the LAD artery from the aorta is located. The position of the ligation depends on the volume of infarction desired. In this study, the LAD artery was ligated $1-2 \mathrm{~mm}$ below the tip of the left atrium in its normal position, which induces roughly $40-50 \%$ ischaemia of the LV. Once the site for ligation had been determined, the curved forceps were used to gently press on the artery a little below the subsequent ligation (to enhance the view of the artery and stabilize the heart). Next, with a tapered needle, a 7-0 silk ligature was passed underneath the LAD coronary artery. For easier and smoother passage the needle was bent in advance to make the curvature rounder. The ligature was then tied with three knots. Occlusion was confirmed by the change of colour (becoming pale) of the anterior wall of the LV. The chest cavity was then closed by bringing together the 4 th and the 5 th ribs with one or two 6-0 nylon sutures (with pressure applied to the chest wall to reduce the volume of free air). The muscles and skin were closed layer-by-layer with 6-0 absorbable and nylon sutures, respectively. The sham-operated mice underwent the same procedure without tying the suture but moving it behind the LAD artery. Both males and females were used and no significant gender difference was noted.

Animal preparation for cardiac function measurements. Anaesthesia was induced by i.p. injection of sodium pentobarbital $\left(50 \mathrm{mg} \mathrm{kg}^{-1}\right)$ and core body temperature was maintained using a heating pad. Animal preparation included: (i) left femoral artery catheterization, (ii) tracheotomy (polyethylene-90 tube to facilitate spontaneous breathing), and (iii) left ventricle conductance catheter introduction through the right carotid artery. Animals were placed in the supine position on the heating pad, before the experimental procedure. Toe pinching test was performed at least every $5 \mathrm{~min}$, and animals who responded received a small dose of sodium pentobarbital $\left(10 \mathrm{mg} \mathrm{kg}^{-1}\right)$ to prevent animal discomfort. Mice were suitable for the experiments if: (1) systemic parameters were within normal

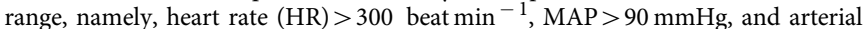
$\mathrm{O}_{2}$ partial pressure $\left(\mathrm{p}_{\mathrm{a}} \mathrm{O}_{2}\right)>80 \mathrm{mmHg}$. Only males were used for these cardiac function measurements. Detailed protocols are described below.

Hypoxia protocol for cardiac function. The anesthetized animals were placed on heating pad within seal acrylic box. As described before for awake animals, the $\mathrm{O}_{2}$ concentrations in the box was changed using compressed air, $15 \% \mathrm{O}_{2}$ balance $\mathrm{N}_{2}$, $10 \% \mathrm{O}_{2}$ balance $\mathrm{N}_{2}$; and $5 \% \mathrm{O}_{2}$ balance $\mathrm{N}_{2}$. Each hypoxic step was maintained for $30 \mathrm{~min}$, and measurements were completed $15 \mathrm{~min}$ after animal's acclimatization. Hypoxia was stopped if blood pressure dropped below $40 \mathrm{mmHg}$, and the animal was excluded from the study.

Systemic parameters. MAP and HR were recorded continuously (MP 150, Biopac System; Santa Barbara, CA, USA).

Cardiac function. The closed chest method was used to study cardiac function. Right common carotid artery was exposed to insert a $1.4 \mathrm{~F}$ pressure-volume conductance catheter (PV catheter; SPR-839, Millar Instruments; Houston, TX, USA). The pressure-volume catheter was advanced passing through the aortic valve into the $\mathrm{LV}^{44}$. At baseline and the end of the experiment, a bolus of $15 \%$ hypertonic saline $(10 \mu \mathrm{l})$ was intravenously injected to determine parallel volume ${ }^{45}$. The pressure and volume signals were continuously acquired (MPVS300, Millar Instruments; Houston, TX, USA and PowerLab 8/30, AD Instruments; Colorado Springs, CO, USA). Left ventricular volume was measured continuously in conductance units (RVU; relative volume unit) and converted to actual blood volume $(\mu \mathrm{l})$ at the end of the experiment.

Cardiac pressure-volume indices. Cardiac function data were analyzed with PVAN software (Millar Instruments, Houston, TX, USA). All cardiac function parameters were averaged from 8 to 12 cardiac cycles at each time point. End systolic pressure $\left(P_{\mathrm{es}}\right)$ was directly measured. Cardiac output $(\mathrm{CO})$, stroke work (SW) and stroke volume (SV) were calculated. VR was calculated as using the MAP divided by the $\mathrm{CO}(\mathrm{VR}=\mathrm{MAP} / \mathrm{CO})$. Oxygen delivery $\left(\mathrm{DO}_{2}\right)$ was calculated as the product of the total $\mathrm{Hb}$ by the $\mathrm{O}_{2}$ carrying capacity of saturated $\mathrm{Hb}\left(1.34 \mathrm{mLO}_{2}\right.$ $\left.\mathrm{g}_{\mathrm{Hb}}\right)$ by the arterial blood $\mathrm{O}_{2}$ saturation and $\mathrm{CO}$

$\left(\mathrm{DO}_{2}=[\mathrm{RBCHb} \times 1.34 \times \mathrm{SA}] \times \mathrm{CO}\right)^{46}$

Hypoxic areas. Hypoxia protocol for awake mice were followed as mentioned earlier. Vital organ hypoxic areas were measured using immunohistochemistry staining for pimonidazole bound to hypoxic zones. Mice received an infusion of the hypoxic marker Hypoxyprobe-1 (pimonidazole $40 \mathrm{mg} \mathrm{kg}^{-1}$ ) and $5 \mathrm{mg} \mathrm{kg}^{-1}$ Hoechst 33342 (Invitrogen Corp., Carlsbad, CA, USA) diluted in PBS (total volume, $100 \mu \mathrm{l}$ ), when they were exposed to $10 \% \mathrm{O}_{2}$. Then, the mice received a second infusion of pimonidazole $\left(40 \mathrm{mg} \mathrm{kg}^{-1}\right)$ and Hoechst $33342\left(5 \mathrm{mg} \mathrm{kg}^{-1}\right)$ diluted in PBS $(100 \mu \mathrm{l})$, when they were exposed $5 \% \mathrm{O}_{2}$. Finally, mice were euthanized and their heart was removed. Tissues were fixed by immersion in formalin for $24 \mathrm{~h}$ at room temperature before transfer to $70 \%(\mathrm{v} / \mathrm{v})$ ethanol. Lastly, tissues were cut into $100-\mu \mathrm{m}$ thick sections.

Pimonidazole immunohistochemistry. Sections were cleaned and rehydrated according to standard procedures. Monoclonal antibody directed against pimonidazole (included in the Hypoxyprobe-1 green kit) was used for immunohistochemical staining of the tissue sections. Fluorescence microscopy was performed using an Olympus BX51WI equipped with a high resolution digital CCD ORCA285 (Hamamatsu Corp., Hamamatsu city, Japan) illuminated with a mercury burner and the appropriate fluorescent cubes (XF100-2 and XF02-2, Omega Optical, Brattleboro, VT). Images for pimonidazole antibody-stained areas and Hoechst were prepared using Wasabi Imaging Software (Hamamatsu Corp). The ratio of pixels stained for pimonidazole in each region, to the total cellular area of the image was calculated. Ten images were analyzed by section, and the results were pooled to determine the mean and standard deviation. To indicate the co-localization of pimonidazole and Hoechst in cell, images were superimposed.

Data availability. All relevant data are included in the figures and/or Supplementary Data, or available from the authors.

\section{References}

1. Frey, N. \& Olson, E. N. Cardiac hypertrophy: the good, the bad, and the ugly. Annu. Rev. Physiol. 65, 45-79 (2003).

2. Giordano, F. J. et al. A cardiac myocyte vascular endothelial growth factor paracrine pathway is required to maintain cardiac function. Proc. Natl Acad. Sci. USA 98, 5780-5785 (2001).

3. Lee, S. H. et al. Early expression of angiogenesis factors in acute myocardial ischemia and infarction. N. Engl. J. Med. 342, 626-633 (2000).

4. Mitsos, S. et al. Therapeutic angiogenesis for myocardial ischemia revisited basic biological concepts and focus on latest clinical trials. Angiogenesis 15, 1-22 (2012).

5. Zachary, I. \& Morgan, R. D. Therapeutic angiogenesis for cardiovascular disease: biological context, challenges, prospects. Heart 97, 181-189 (2011).

6. van der Laan, A. M., Piek, J. J. \& van Royen, N. Targeting angiogenesis to restore the microcirculation after reperfused MI. Nat. Rev. Cardiol. 6, 515-523 (2009).

7. Potente, M., Gerhardt, H. \& Carmeliet, P. Basic and therapeutic aspects of angiogenesis. Cell 146, 873-887 (2011).

8. Gude, N. A. et al. Activation of Notch-mediated protective signaling in the myocardium. Circ. Res. 102, 1025-1035 (2008).

9. Croquelois, A. et al. Control of the adaptive response of the heart to stress via the Notch1 receptor pathway. J. Exp. Med. 205, 3173-3185 (2008).

10. Kopan, R. \& Ilagan, M. X. The canonical Notch signaling pathway: unfolding the activation mechanism. Cell 137, 216-233 (2009).

11. Kao, H. Y. et al. A histone deacetylase corepressor complex regulates the Notch signal transduction pathway. Genes Dev. 12, 2269-2277 (1998).

12. Liefke, R. et al. Histone demethylase KDM5A is an integral part of the core Notch-RBP-J repressor complex. Genes Dev. 24, 590-601 (2010).

13. Castel, D. et al. Dynamic binding of RBPJ is determined by Notch signaling status. Genes Dev. 27, 1059-1071 (2013).

14. Sakai, T. et al. Loss of immunostaining of the RBP-J kappa transcription factor upon F9 cell differentiation induced by retinoic acid. J. Biochem. 118, 621-628 (1995).

15. Gillers, B. S. et al. Canonical wnt signaling regulates atrioventricular junction programming and electrophysiological properties. Circ. Res. 116, 398-406 (2015) 
16. Lei, L. et al. Hypoxia-inducible factor-dependent degeneration, failure, and malignant transformation of the heart in the absence of the von Hippel-Lindau protein. Mol. Cell Biol. 28, 3790-3803 (2008).

17. Holscher, M. et al. Unfavourable consequences of chronic cardiac HIF-1alpha stabilization. Cardiovasc. Res. 94, 77-86 (2012).

18. Oswald, F. et al. RBP-Jkappa/SHARP recruits CtIP/CtBP corepressors to silence Notch target genes. Mol. Cell Biol. 25, 10379-10390 (2005).

19. Sohal, D. S. et al. Temporally regulated and tissue-specific gene manipulations in the adult and embryonic heart using a tamoxifen-inducible Cre protein. Circ. Res. 89, 20-25 (2001).

20. Gupta, R., Tongers, J. \& Losordo, D. W. Human studies of angiogenic gene therapy. Circ. Res. 105, 724-736 (2009).

21. Sano, M. et al. p53-induced inhibition of Hif-1 causes cardiac dysfunction during pressure overload. Nature 446, 444-448 (2007).

22. Wettstein, D. A., Turner, D. L. \& Kintner, C. The Xenopus homolog of Drosophila suppressor of hairless mediates Notch signaling during primary neurogenesis. Development 124, 693-702 (1997).

23. Kulic, I. et al. Loss of the Notch effector RBPJ promotes tumorigenesis. J. Exp. Med. 212, 37-52 (2015)

24. Borggrefe, T. \& Oswald, F. Keeping notch target genes off: a CSL corepressor caught in the act. Structure. 22, 3-5 (2014).

25. Johnson, J. E. \& Macdonald, R. J. Notch-independent functions of CSL. Curr. Top. Dev. Biol. 97, 55-74 (2011).

26. Kratsios, P. et al. Distinct roles for cell-autonomous Notch signaling in cardiomyocytes of the embryonic and adult heart. Circ. Res. 106, 559-572 (2009).

27. Kolar, F. \& Ostadal, B. Molecular mechanisms of cardiac protection by adaptation to chronic hypoxia. Physiol. Res. 53, S3-S13 (2004).

28. Han, H. et al. Inducible gene knockout of transcription factor recombination signal binding protein-J reveals its essential role in T versus B lineage decision. Int. Immunol. 14, 637-645 (2002).

29. Hsieh, P. C. et al. Evidence from a genetic fate-mapping study that stem cells refresh adult mammalian cardiomyocytes after injury. Nat. Med. 13, 970-974 (2007).

30. Hamaguchi, Y. et al. Biochemical and immunological characterization of the DNA binding protein (RBP-J kappa) to mouse J kappa recombination signa sequence. J. Biochem. 112, 314-320 (1992).

31. Wei, K. et al. Developmental origin of age-related coronary artery disease. Cardiovasc. Res. 107, 287-294 (2015).

32. Manders, E. E. M., Verbeek, F. J. \& Aten, J. A. Measurement of co-localisation of objects in dual colour confocal images. J. Microscopy 169, 375-382 (1993).

33. O'Connell, T. D., Rodrigo, M. C. \& Simpson, P. C. Isolation and culture of adult mouse cardiac myocytes. Methods Mol. Biol. 357, 271-296 (2007).

34. Campa, V. M. et al. Notch activates cell cycle reentry and progression in quiescent cardiomyocytes. J. Cell Biol. 183, 129-141 (2008).

35. Prigozhina, N. L. et al. Characterization of a novel angiogenic model based on stable, fluorescently labelled endothelial cell lines amenable to scale-up for high content screening. Biol. Cell 103, 467-481 (2011).

36. Livak, K. J. \& Schmittgen, T. D. Analysis of relative gene expression data using real-time quantitative PCR and the 2(-Delta Delta C(T)) Method. Methods 25, 402-408 (2001).

37. Shima, D. T. et al. The mouse gene for vascular endothelial growth factor. Genomic structure, definition of the transcriptional unit, and characterization of transcriptional and post-transcriptional regulatory sequences. J. Biol. Chem. 271, 3877-3883 (1996).

38. Kondo, K., Klco, J., Nakamura, E., Lechpammer, M. \& Kaelin, Jr W. G. Inhibition of HIF is necessary for tumor suppression by the von Hippel-Lindau protein. Cancer Cell 1, 237-246 (2002).

39. Simone, C. et al. p38 pathway targets SWI-SNF chromatin-remodeling complex to muscle-specific loci. Nat. Genet. 36, 738-743 (2004).

40. Rockman, H. A. et al. Control of myocardial contractile function by the level of beta-adrenergic receptor kinase 1 in gene-targeted mice. J. Biol. Chem. 273, 18180-18184 (1998)
41. Rockman, H. A. et al. Expression of a beta-adrenergic receptor kinase 1 inhibitor prevents the development of myocardial failure in gene-targeted mice. Proc. Natl Acad. Sci. USA 95, 7000-7005 (1998).

42. Tanaka, N. et al. Transthoracic echocardiography in models of cardiac disease in the mouse. Circulation 94, 1109-1117 (1996).

43. Tarnavski, O. et al. Mouse cardiac surgery: comprehensive techniques for the generation of mouse models of human diseases and their application for genomic studies. Physiol. Genomics 16, 349-360 (2004).

44. Pacher, P., Nagayama, T., Mukhopadhyay, P., Batkai, S. \& Kass, D. A. Measurement of cardiac function using pressure-volume conductance catheter technique in mice and rats. Nat. Protoc. 3, 1422-1434 (2008).

45. Baan, J. et al. Continuous measurement of left ventricular volume in animals and humans by conductance catheter. Circulation 70, 812-823 (1984).

46. Raab, S., Thein, E., Harris, A. G. \& Messmer, K. A new sample-processing unit for the fluorescent microsphere method. Am. J. Physiol. 276, H1801-H1806 (1999).

\section{Acknowledgements}

We thank Nancy Dalton and Yusu Gu for performing TAC and echocardiography experiments, Professor T. Honjo (Kyoto University) for providing the anti-RBPJ T6709 antibody and the RBPJ floxed mice, and Ana Maria Manso and Robert S. Ross (San Diego Veterans Administration Hospital and University of California, San Diego) for tissue samples. This work was supported by NIH R01 HL083463, R01 HL128072, R01 HL113601 and R01 HL108176 and the Sanford Children's Health Center to M.M.; NIH R01 HL065484 and R01 HL086879 to P.R-L. and P01 HL098053 to M.M./P.R-L.; NHLBI K08 HL107449 and AHA Grant in Aid 14GRNT19510011 to S.R. who holds a Career Award for Medical Scientists from the Burroughs Wellcome Fund; and by NIH P01 HL110900, R01 HL52684 and R56 HL123015 to P.C.; CIRM Postdoctoral Fellowship and fellowships from of the Italian Ministry of Research and Education and the Italian Society of Cardiology to M.C.S., and an American Heart Association 11SDG761017 SDG Grant to R.D-T. Core services at Sanford-Burnham-Prebys were supported by P30 CA030199.

\section{Author contributions}

R.D-T., M.C.S., A.M., E.M., D.T. and P.C. performed and interpreted experiments. S.R. developed the iNICD mouse model. K.P. supervised the TAC experiments and provided expert consultation. R.D-T., P.R-L. and M.M. designed the study and prepared the manuscript.

\section{Additional information}

Supplementary Information accompanies this paper at http://www.nature.com/ naturecommunications

Competing financial interests: The authors declare no competing financial interests

Reprints and permission information is available online at http://npg.nature.com/ reprintsandpermissions/

How to cite this article: Díaz-Trelles, R. et al. Notch-independent RBPJ controls angiogenesis in the adult heart. Nat. Commun. 7:12088 doi: 10.1038/ncomms12088 (2016).

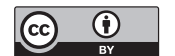

This work is licensed under a Creative Commons Attribution 4.0 International License. The images or other third party material in this article are included in the article's Creative Commons license, unless indicated otherwise in the credit line; if the material is not included under the Creative Commons license, users will need to obtain permission from the license holder to reproduce the material To view a copy of this license, visit http://creativecommons.org/licenses/by/4.0/

C) The Author(s) 2016 